\title{
LA IMPORTANCIA DE LA SALUD MENTAL EN EL MUNDO LABORAL
}

Maria José Bustamante Zárate

Facultad de Negocios, Gestión y Sostenibilidad Estudiante, Programa de Negocios Internacionales mabustamante8@poligran.edu.co Juliana Gómez Rojas Facultad de Negocios, Gestión y Sostenibilidad ngomezr@poligran.edu.co

Daniel Felipe Tenjo

Facultad de Negocios, Gestión y Sostenibilidad Estudiante, Programa de Negocios Internacionales datenjoc@poligran.edu.co

Daniela Navarro Bustamante

Facultad de Negocios, Gestión y Sostenibilidad Estudiante, Programa de Administración de Empresas Danavarro4@poligran.edu.co Jorge Fernando Lara

Facultad de Negocios, Gestión y Sostenibilidad Estudiante, Programa de Administración de Empresas jolarach@poligran.edu.co 


\section{Resumen}

El presente artículo nos muestra las claves principales por las cuales una persona asciende en el ámbito laboral y es más productiva.

Para esto, traeremos las respectivas definiciones de salud mental, ascenso laboral y empresa, que nos ayudarán a hacer conexiones relacionadas con el tema a tratar; haremos un análisis relacionando el éxito de una persona en el ambiente laboral con su salud psicológica, basándonos en la teoría estructuralista de la administración, en su definición y en sus respectivas características.

También, retroalimentamos frente a las pautas que las empresas deberían implementar para que sus empleados tengan un espacio laboral óptimo, en donde se sientan cómodos y de cierta manera felices, para que así puedan desarrollar sus labores sin ningún inconveniente.

Palabras claves: Salud mental, laboral, empleo, desempeño, producción

\section{Introducción}

El presente trabajo muestra como su nombre lo indica, la importancia de la salud mental en el ámbito laboral y cómo este afecta positiva o negativamente a las personas al momento de desempeñar alguna labor, también vamos a poder ver desde diferentes puntos de vista cómo estas enfermedades laborales ya sean físicas o mentales son perjudiciales para el empleado y la empresa, ya que si el empleado baja su rendimiento la empresa se ve afectada en su producción y a largo plazo en su economía.

Mostraremos como es la composición de las empresas, también datos sobre la economía colombiana y sus pocas oportunidades laborales que afecta negativamente a los empleados, ya que al tener pocas oportunidades ingresan a trabajar donde les toca y esto a largo plazo afecta su nivel emocional, por otro lado las empresas se aprovechan de esto y ponen muchas 
labores o tareas a los pocos empleados que tienen, afectando así su vida cotidiana y su nivel emocional por tratar de cumplir con todas las labores.

Posteriormente, se muestran formas de tratar las enfermedades y cómo las diferentes entidades prestadoras de salud muestran datos de las personas afectadas por dichas enfermedades.

\section{Marco teórico}

\section{Salud mental}

La salud mental es el balance entre una persona y su entorno sociocultural en el cual, dicho por la OMS (Organización mundial de la salud), se encuentra bienestar físico, mental y social que no solamente se da por la ausencia de enfermedades. Esto le proporciona a la persona calidad de vida, la cual le permite tener participación laboral, intelectual y de relaciones de una manera óptima.

La salud mental nos ayuda en muchísimas pautas de nuestra vida, desde hacer frente a un problema que se nos presenta, hasta trabajar de manera más productiva en nuestro empleo haciéndonos alcanzar nuestro mayor potencial.

También es un hecho que cuando estamos mentalmente sanos, se refleja en nuestro cuerpo, y nos acarrea buena salud física.

Una enfermedad mental es un padecimiento grave que puede afectar el comportamiento y la manera de pensar de una persona. Puede darse de manera ocasional o con una duración prolongada. Impide que una persona se relacione de una manera óptima.

Esta afección es tan común que más del $50 \%$ de todos los estadounidenses son diagnosticados con un trastorno mental a lo largo de su vida.

Sin embargo, existen tratamientos que permiten que las personas con este tipo de trastornos lleven una vida plena, y en muchos casos estos desaparecen por completo.

\section{Empresa}


Es definida como la unidad formada por bienes materiales y personas que tienen como propósitos producir o prestar un servicio.

Clasificación de empresas según la actividad económica que desarrolla:

1. Sector primario: se encarga de la creación de los bienes obteniendo los recursos de la naturaleza (agrícolas, ganaderas, pesqueras, mineras, etc.).

2. Sector secundario: se basa en transformar físicamente los bienes en otros más útiles para su uso. En este grupo se encuentran las empresas industriales y de construcción. Ejemplo: tienes una naranja y la conviertes en jugo de naranja, se empaqueta y se vende.

3. Sector terciario: (servicios y comercio), se encuentran actividades de diversa naturaleza, como comerciales, transporte, turismo, etc.

\section{Micro, pequeña y mediana empresa en Colombia.}

La clasificación de las empresas en Colombia está dada así:

Gráfica 1. Clasificación de las empresas en Colombia según sus activos totales y el número de empleados.

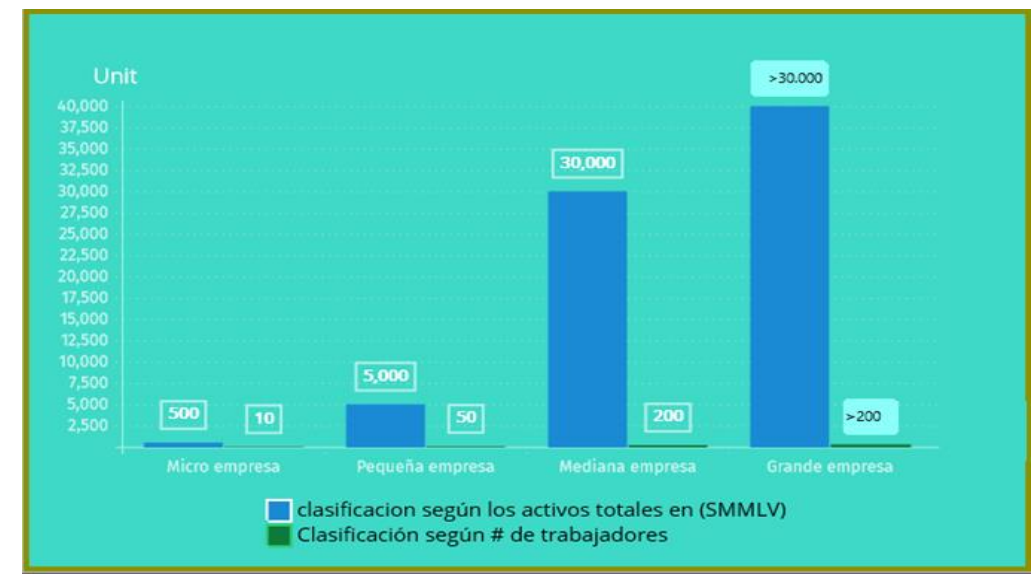

Fuente: Elaboración propia.

Para las empresas que combinen el número de trabajadores y el valor de los activos totales, va a prevalecer el valor de los activos totales. 


\section{* Ley 905 de 2004}

\section{La situación de empleo en el país}

Colombia es un país que tiene acuerdos comerciales con 58 países. Es el tercer país en América Latina (superado por México y Chile) en este aspecto. Lamentablemente este acontecimiento se ha traducido en la generación de más y mejores empleos.

Aportes de cada sector al empleo nacional en el 2018:

- El sector minero-energético participó con 0,9\% del total de empleo nacional (211.000 puestos).

- La industria manufacturera aportó en un 12\% (aprox 2,7 millones de puestos).

- El sector agrícola aportó un 16,7\% (3,8 millones de puestos).

- El sector de comercio, hoteles y restaurantes aportó el 26,8\% (unos 6 millones).

\section{Ascenso laboral}

Como lo indica la palabra un ascenso se refiere a un aumento de cargo en la labor que desarrolla una persona en una empresa. Este aumento genera beneficios para el trabajador, desde un aumento de sueldo, hasta más reconocimiento frente a todos los directivos en la empresa.

\section{EFECTOS DE LA SALUD PSICOLÓGICA EN EL ÁMBITO LABORAL.}

Cuando se habla de salud laboral, se piensa en cómo afecta el trabajo a la salud corporal y las pautas para la prevención de riesgos laborales se centran en la seguridad física. Pero ¿qué pasa con la mental? Cada profesional pasa más de un tercio del día en su puesto de trabajo, por lo que el bienestar psicológico resulta crucial para lograr un mejor rendimiento.

El trabajo, en muchas ocasiones puede ser considerado como una fuente de satisfacción y/o realización personal. Por el contrario, en el desempleo (Wad Dell y Burton, 2006), el ámbito laboral puede tener un visible efecto negativo sobre el bienestar y la salud mental de los trabajadores. 
Dicho por la OMS, las enfermedades psicológicas y emocionales son un problema de todos los días en el trabajo, ya que los empleados se ven a afectados por diferentes factores, estos a su vez generan un déficit en ellos causando disminución en su productividad, estos trastornos como algunas entidades los llaman varían según la edad y el género, pero el más general es el estrés, este afecta a casi la mayoría de la población, tiene diferentes fuentes, como lo son, problemas familiares, problemas amorosos, superación y emocionales, así mismo este si no se trata adecuadamente lleva problemas tanto físicos como psicológicos.

La primera vez que se utilizó este término fue en la década de los 70 para refiriéndose a los problemas de cansancio y fatiga profesional de los empleados de los servicios sociosanitarios (trabajadores del área de salud mental, servicios sociales, profesionales educativos).

\section{Metodología}

\section{Cualitativo}

Existe una técnica de recogida de datos llamada grupo focal que se utiliza en investigaciones de mercado a comienzos del año 1950. Empieza a ser atractivo para investigadores de otras áreas del conocimiento, como lo es el área de la salud, ya que se empezaron a dar muchas enfermedades en sus trabajadores a nivel mental, entre los cuales iban incluidos suicidios. Esta técnica es de suma importancia para así darle una utilización en ámbitos de la salud con la perspectiva de lo social al proponer el estudio de representaciones y relaciones de los diferentes grupos profesionales del área, de diferentes procesos de población y trabajos.

Este estudio tiene como función exponer los aspectos teóricos que hacen posible la utilización de la técnica de un conjunto focal en la recolección de aspectos cualitativos. Esto es esencial para tratar las cuestiones de la salud en el ambiente de trabajo, bajo el ámbito social, ya que se plantea un estudio de relaciones de los diferentes grupos profesionales en el área, para diversos procesos de trabajo e incluso la posibilidad de determinar obligaciones para la salud en la población. Para el manejo de este sistema el examinador debe exigir una organización sumamente planificada de etapas que integran esta técnica. Este debe estar atento y proveer a instante la recopilación de información, para tener total claridad al momento de agrupar a los integrantes en base a un tema específico, y de esta forma cumplir los objetivos de la investigación ofertada. 
Para la utilización de este método del grupo focal es de suma importancia considerar bases epistemológicas y metodológicas que se fundamentan en la discusión e interacción, incluidas en la tradición dialéctica, encargándose de la construcción del conocimiento en espacios de intersubjetividad.

La técnica se encarga de apreciar la interacción y la estimulación del diálogo con respecto a temas que llegan a ser incómodos, que tienden a adentrarse en informaciones para captar comportamientos en determinado contexto cultural.

\section{EL CRECIMIENTO DE LAS PERSONAS EN LAS ORGANIZACIONES.}

En el departamento de recursos humanos de las organizaciones o empresas, se tiene como objetivo analizar el comportamiento de sus empleados, donde el desempeño es un fenómeno que determina la productividad de los mismos, dejando cierta satisfacción, llevando a una estabilidad laboral, donde no habrá rotación y ausentismo por parte de los empleados; anotando a una solidez, eficiencia y permanencia organizacional.

También fundamentándose hacia un entorno más competitivo y globalizado, ya que las organizaciones se enfrentan en la actualidad, a la constante lucha por estar posicionados en un mercado, provocando en ellos una esclavitud, debida a la filosofía de satisfacción que debe de llevar una empresa, para poder así llegar al reconocimiento por parte de una sociedad consumista.

Por tratarse de temas psicológicos, en específico, lo psicosocial es de mucha importancia, investigar la psicología del trabajo y el comportamiento del ser humano dentro de una organización. Por lo consecuente se implementan estrategias, las cuales puedan mejorar la eficiencia dentro del área de trabajo, sin dejar a un lado el concepto humanista que debe de emplear una empresa, para que sus empleados puedan desenvolverse de la mejor manera en su campo laboral.

\section{Análisis de los datos}

Se determinó por la previa recolección de información acerca de la salud mental de un trabajador dentro de una organización, de cómo ha habido una evolución por parte de las estrategias que el humano ha podido estructurar, para que su desenvolvimiento dentro de una 
empresa sea sano para su salud mental como trabajador y como ser humano. Sin perder de vista el tamaño, que tiene la empresa, en la cual esa persona está empleando.

Evaluando diferentes puntos de vistas, se puede observar que, el entorno de trabajo de un empleado es de suma importancia, debido a que es el reflejo de un posible resultado, entonces aquí hay una dualidad entre la "buena y la mala" área de trabajo, porque el descuido y el obsesivo cuidado de la misma, puede ser un factor que afecte al empleador inconscientemente o conscientemente.

Esta problemática en la mayoría de los empleados no es vista conscientemente, debido a que hay una constante culpabilidad por parte del mismo, porque no hacen un análisis general de dicha situación, dejando una sola mirada introspectiva, sin darse cuenta que están generando un desánimo y posibles efectos psicológicos, que afectan su rendimiento laboral.

Por eso constantes investigaciones por parte de entidades, asociadas con la salud mental y el trabajo, han demostrado que debe haber un debido proceso llamado equilibrio, donde las perspectivas internas y externas de una empresa, se deben de llevar a cabo continuamente, porque así abren un campo lleno de posibles estrategias viables para una solución colectiva o individual, dependiendo el caso que se quiera atender.

Por eso grandes organizaciones, se caracterizan por tener este pilar de la salud mental, muy alto, porque su mismo tamaño, reconocimiento y calidad, demanda una buena estructuración y un control dentro de la misma, porque para ellos es de suma importancia la salud mental, emocional, salud psicológica, etc., de sus trabajadores, debido que son los encargados de resaltar la calidad de aquel producto o servicio, brindado por ellos.

Por otra parte, se resalta esas ciertas estrategias que, con la evolución de los mercados, se han vuelto obsoletas o no rentables, y una de estas es el incremento salarial.

A cambio de más horas de trabajo, actualmente se ha demostrado que entre más el ser humano elabore, hay un incremento y hasta surgimiento de enfermedades que afectan la estabilidad emocional del humano, porque constantemente se ha hecho la dualidad de vidas dentro de este mundo, donde una innatamente es de suma importancia para supervivencia humana y la otra es una que el hombre ha creado con base de la anterior, donde está en la actualidad ha demostrado que es más importante, porque sin una vida laboral, no hay donde disfrutar o mismo sostenerse en la vida "privada". 
Por eso el humano ha luchado constantemente para demostrarse a sí mismo que es capaz de hacer todo, sin tener en cuenta su salud mental y física.

Este tipo de estrategias deben de tener un seguimiento a detalle, para que no se vuelvan un problema global dentro de la organización. El humano no puede permitir que la competencia dentro de una empresa, sea la destrucción de su estabilidad en el diario vivir. Por consiguiente, el humano debe de tener un equilibrio en su vida laboral, donde ese mundo que él mismo se vio obligado a vivir, haya control y dirección de lo que es sano para su salud mental y física.

\section{Conclusiones}

podríamos decir que el ascenso laboral es un incentivo emocional traído desde el siglo XIX para hacer que los trabajadores se sientan más motivados en sus labores, trabajen con más ánimos y ganas, y por lo tanto sean más productivos.

También podemos concluir que las enfermedades psicológicas y emocionales son un problema de todos los días y se da en todas las empresas. Los empleados se ven a afectados por diferentes factores los cuales generan problemas causando disminución en su productividad.

Además, estos trastornos varían según la edad y el género, pero el más general es el estrés, que afecta a casi la mayoría de la población. Se puede dar por problemas familiares, problemas amorosos, y por supuesto, problemas laborales. así mismo este si no se trata adecuadamente lleva problemas tanto físicos como psicológicos.

algunos de los indicadores que nos muestran que una persona no se encuentra bien en cuanto a su salud mental son: Agotamiento emocional, despersonalización, realización personalización, en las cuales sus consecuencias pueden ser: aburrimiento y estrés, crisis en el desarrollo de la carrera profesional, sobrecarga profesional, falta de estimulación y aislamiento. Todo esto afecta directamente al desarrollo de un profesional en el área en la que se especializa en determinada empresa.

Como resultado de lo anterior se puede inferir que las empresas son las primeras que deben poner en la mira este tema, ya que al buscar soluciones o facilitar la vida de los trabajadores y que estos no tengan enfermedades emocionales, serían los más beneficiados. 
Por otro lado, al comparar las diferentes opiniones de las entidades, se puede indicar que la salud mental de los trabajadores y todo lo que la afecta tiene cura y existen diferentes tratamientos para ayudar.

De esta manera mostramos como la salud mental de los empleados es un factor muy importante el cual las empresas deben poner cuidado y debe ser uno de sus pilares para que la empresa crezca y mejore su productividad.

Se puede añadir, que debe de haber control y dirección de lo que es sano para la salud mental del trabajador, teniendo en cuenta su entorno, la carga laboral, el puesto que ejerce dentro la organización. Por se opta por estrategias, las cuales sean rentables según la problemática o carga que esté sobrepasando la empresa.

Teniendo como resultado, la competencia dentro del mercado es un factor que también puede afectar el estado emocional de los trabajadores, además de toda la empresa, debido a los resultados que debe de sostener, para que no pierda el reconocimiento por parte de los clientes y de los mismos proveedores de su producto o servicio. Generan preocupación y angustia constantemente, por eso el manejo de las estrategias psicológicas dentro del ámbito laboral son fundamento para continuar con ese régimen común de producción, que hacen a la compañía solidaria y fuerte.

Creemos que, para obtener organización, eficaz y de una excelente calidad, para la atención de la salud mental es de indispensable un Servicio de Planificación, Gestión y una Financiación, potenciando y regulando los mecanismos de coordinación entre los dispositivos asistenciales de la red de salud mental y la coordinación con los recursos que son para la comunidad.

La salud mental es un tema que hoy por hoy, atropella a la mayoría de las personas, ya sea por problemas familiares, entorno laboral, etc, propiciando a los empleados incluso a tomar decisiones drásticas como el suicidio, por ende, una organización siempre debe estar abierta y darles seguimiento a sus trabajadores, para garantizar su estabilidad emocional, que consigo traerán excelentes beneficios a la compañía.

El síndrome de burnout es real, y es fatal para quienes no lo acepten, para aquellos que logran agotarse físicamente, generando ataques de pánico en momentos inesperados. Es una enfermedad que ataca de manera recurrente, y en una mayoría a las personas con una carga 
grande de actividades, las cuales generan un estrés crónico en ellas, teniendo ataques de pánico, dificultad para concentrarse, agotamiento mental y físico. Por consiguiente, es una enfermedad la cual debe de tener atención por parte de la empresa y de ayuda médica. Por eso se debe de buscar una solución, para que deje de verse como una paranoia por parte de los empleadores, sino como lo que realmente es una enfermedad. 


\section{REFERENCIAS BIBLIOGRÁFICAS}

- Kellaway, L. (2013). La invención del ascenso laboral BBC. Recuperado de: https://www.bbc.com/mundo/noticias/2013/08/130808_serie_oficina_invencion_asce nso_laboral_finde_jrg.shtml

- El ascenso dentro de una empresa. (2005).20 minutos. Recuperado de:

- https://www.20minutos.es/noticia/3711/0/ASCENSO/EMPRESA/DENTRO/

- Ranstad. (2019). Cómo afecta la psicología en el trabajo. Recuperado de:https://www.randstad.es/tendencias360/como-afecta-la-psicologia-en-el-trabajo/

- Castillo,S.(2001).Scielo.Recuperado de:https://www.scielo.sa.cr/scielo.php?pid=S140900152001000100004\&script=sci_arttext

- Clasificación de las empresas colombianas. (2019). Encolombia. Recuperado de: https://encolombia.com/economia/economiacolombiana/empturisticos/clasificaicondelasempresascolombianas/

- Organización mundial de la salud. (2019). salud mental en el lugar de trabajo. Recuperado de: https://www.who.int/mental_health/in_the_workplace/es/

- Infocop. (2017). El papel del bienestar en el ámbito laboral: fundamentos y aportaciones desde la Psicología. Recuperado de: http://www.infocop.es/view_article.asp?id=7038

- Dias CA. Grupo focal: técnica de coleta de dados em pesquisas qualitativas. Informação e Sociedade. Estudos 2000; 10(2): 1-12. Recuperado de: http://scielo.isciii.es/scielo.php?script=sci_arttext\&pid=S1132-12962013000100016

- Minayo MCS. O desafio do conhecimento: pesquisa qualitativa em saúde. 5 ed. São Paulo: Hucitec; 2000. Recuperado de: http://scielo.isciii.es/scielo.php?script=sci_arttext\&pid=S1132-12962013000100016

- Gatti BA. Grupo focal na pesquisa em ciências sociais e humanas. Brasília: Líber Livro; 2005. Recuperado de:

- http://scielo.isciii.es/scielo.php?script=sci_arttext\&pid=S1132-12962013000100016

- Gomes AA. Usos e possibilidades do grupo focal e outras alternativas metodológicas. Enfoques 2003; 2(1): 1-8. Recuperado 
de:http://scielo.isciii.es/scielo.php?script=sci_arttext\&pid=S113212962013000100016

- Baron, R. M. \& Kenny, D. A. (1986). The moderator-mediator variable. Recuperado de: distinction in social psychological research: Conceptual, strategic and statistical considerations. Journal of Personality and Social Psychology, 51(6), 1173-1182. Recuperado de:https://www.redalyc.org/pdf/647/64730432009.pdf

- Bond, F., Flaxman, P. \& Bunce, D. (2008). The influence of psychological flexibility on work redesign: Mediated moderation of a work reorganization intervention. Journal of Applied Psychology, 93(3), 645-654. Recuperado de: https://www.redalyc.org/pdf/647/64730432009.pdf

- Waddell, G. y Burton, A.K. Is work good for your health and well-being? Department of Work and Pensions, UK. 2006. London: TSO

- Parra, M. (2004). Promoción y protección de la salud mental en el trabajo: Análisis conceptual y sugerencias de acción. Recuperado de: https://pdfs.semanticscholar.org/1aa9/cf01e5d01c94cb624e1cec71e58ad6b0b0a9.pdf

- El Espectador. (2017).Empresas, a vigilar la salud mental. Recuperado de: https://www.elespectador.com/noticias/salud/empresas-vigilar-la-salud-mentalarticulo-717412

- La República. (2019). La salud mental en las compañías, un tema al que le deben prestar atención.recuperado de:https://www.larepublica.co/salud-ejecutiva/el-impactoque-tiene-la-salud-mental-en-las-companias-2892311

- Prevención integral. (2015).colombia: La salud mental de los trabajadores,prioridad del ministerio de Trabajo. Recuperado de: https://www.prevencionintegral.com/actualidad/noticias/2017/07/25/colombia-saludmental-trabajadores-prioridad-ministerio-trabajo

- Bracco, Valdez, Wakeham \& Velázquez. (2019). Síndrome de agotamiento profesionales y trabajadores penitenciarios peruanos. Una mirada cualitativa a los factores institucionales y sociales. Recuperado de: https://dialnet.unirioja.es/servlet/articulo?codigo=6923197 